Z Rheumatol 2020 · 79:200-202

https://doi.org/10.1007/s00393-020-00749-8

Online publiziert: 10. Februar 2020

(c) Der/die Autor(en) 2020

\section{Redaktion}

K. Albrecht, Berlin

E. Edelmann, Bad Aibling

W. Graninger, Graz

W. Mau, Halle/Saale

F. Schuch, Erlangen

P.M. Villiger, Bern

\author{
R. Hasseli' ${ }^{1}$ S. Pfeiffer ${ }^{2} \cdot$ J. Kappesser ${ }^{2} \cdot$ C. Hermann ${ }^{2} \cdot$ K. Richter-Bastian ${ }^{3} \cdot$ \\ T. Sattler ${ }^{1} \cdot$ M. Tschernatsch ${ }^{4} \cdot$ U. Hoffmann ${ }^{5} \cdot$ U. Müller-Ladner ${ }^{1} \cdot$ U. Lange ${ }^{1}$ \\ 'Abteilung für Rheumatologie und Klinische Immunologie, Justus-Liebig-Universität Gießen, Campus \\ Kerckhoff, Bad Nauheim, Deutschland \\ ${ }^{2}$ Klinische Psychologie und Psychotherapie, Justus-Liebig-Universität, Gießen, Deutschland \\ ${ }^{3}$ Zentrum für unerkannte und seltene Erkrankungen, Universitätsklinikum Gießen - Standort Marburg, \\ Marburg, Deutschland \\ ${ }^{4}$ Neurologie, MVZ am Hochwald, Gesundheitszentrum Wetterau, Bad Nauheim, Deutschland \\ ${ }^{5}$ Logopädische Praxis, Friedberg-Ossenheim, Deutschland
}

\title{
Modellprojekt zur interdisziplinären universitären Lehre - Studierende der Medizin und der Psychologie lernen erstmals gemeinsam
}

Die medizinische Versorgung chronisch erkrankter Patienten ist komplex und erfordert eine interdisziplinäre Zusammenarbeit. Vor allem im Bereich der internistischen Rheumatologie ist eine interdisziplinäre Arbeit unerlässlich, um die komplexen somatischen und psychosozialen Aspekte einer chronischen Erkrankung zu berücksichtigen. Dennoch kommen die Aspekte der interprofessionellen Arbeit im Studium und in der Ausbildung von Ärzt*innen und Psycholog*innen zu kurz und werden dadurch im klinischen Alltag wenig gelebt. Eine unterschiedliche fachspezifische Sprache fördert zusätzlich Ressentiments und Vorurteile zwischen den Professionen, was letztlich zu einer Verschlechterung der Patientenversorgung führt.

Interprofessionelles Verständnis mit dem Ziel einer zunehmenden Interdisziplinarität in der Versorgung von Patienten sollte möglichst frühzeitig, also bereits im Studium vermittelt werden. Hierfür hat sich ein Team des Fachbereichs Medizin der Justus-LiebigUniversität Gießen unter der Leitung von Dr. Rebecca Hasseli und Prof. Dr. Uwe Lange (Abt. für Rheumatologie am Campus Kerckhoff) und der klinischen
Psychologie \& Psychotherapie unter der Leitung von Silas Pfeiffer, Dr. Judith Kappesser und Frau Prof. Dr. Christiane Hermann gebildet und ein neues Lehrkonzept konzipiert, um Psychologie- und Medizinstudierende erstmalig gemeinsam zu unterrichten. Über einen Zeitraum von 1 Woche erhalten die Studierenden Einblicke in die Bereiche der Physio- und Schmerz(psycho)therapie, Neurologie und Logopädie. Durch praktische Übungen wird das theoretische Wissen in die Praxis übertragen und gefestigt. Die Teilnehmer*innen konn- ten in Tandemteams (je 1 Mediziner*in und 1 Psychologe ${ }^{\star}$ in) gemeinsam $\mathrm{Pa}$ tient ${ }^{*}$ innen befragen und untersuchen und versuchen, die körperlichen und psychischen Beschwerden der Patienten herauszuarbeiten und ganzheitliche Behandlungsangebote zu erarbeiten.

Erstmalig konnte das Lehrkonzept unter dem Titel „Interdisziplinarität in der Patientenversorgung “ in Form eines Wahlfachs zu Beginn des Wintersemesters 2019/20 angeboten werden und stieß bereits in der Anmeldephase auf eine besonders positive Resonanz bei den

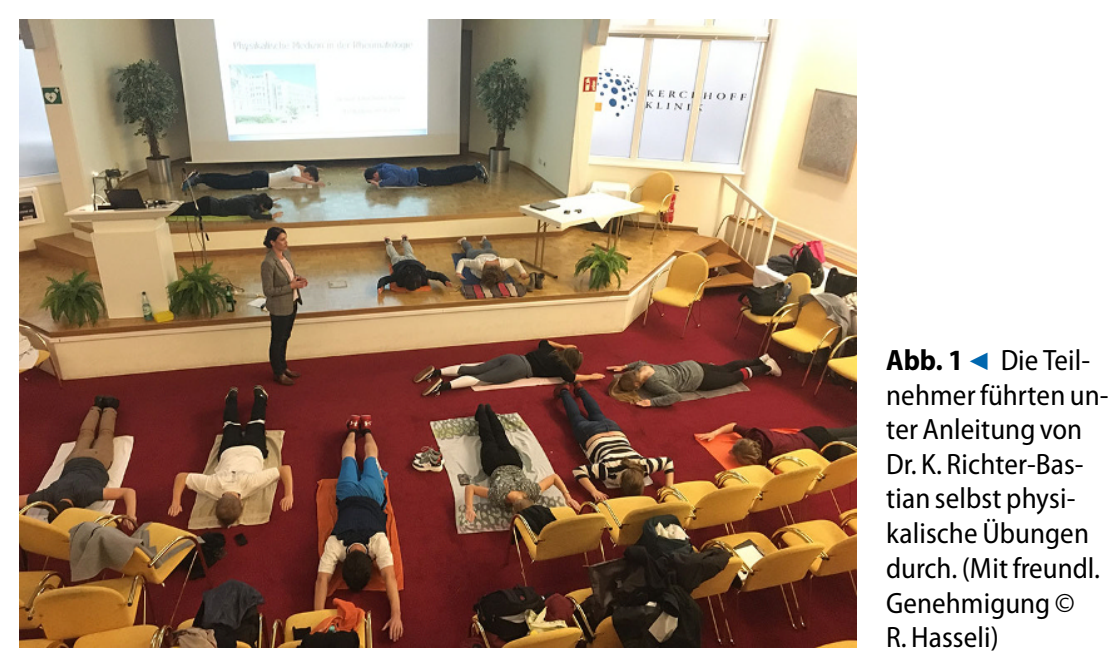


Studierenden. Durch eine Auswahl an erfahrenen Referenten aus der Praxis und Klinik der Physiotherapie, Logopädie, Schmerztherapie und Neurologie erhielten die Teilnehmer in Theorie und Eigenerfahrung einen Einblick in die jeweiligen Bereiche. Dadurch erlernten die Teilnehmer, die Erkrankungen der Patienten aus dem Blickwinkel verschiedener Fachdisziplinen zu betrachten und Behandlungsmöglichkeiten abzuleiten. Diese Art der Lehre gemeinsam mit der Kooperation der Psychologie und Medizin stellt eine Innovation dar und soll auch Anstoß sein, dieses Konzept umzusetzen und weiterzuentwickeln.

An den ersten beiden Tagen wurde im Rahmen von Referaten, die die Teilnehmer*innen in gemischten Teams vorgetragen haben, ein Einblick in die Psychotherapie und entzündlich rheumatische Erkrankungen gegeben. Mithilfe von Gruppenarbeiten wurde die Theorie zudem in die Praxis übertragen. Hierbei wurden z.B. Imaginationsübungen mit der Gruppe erprobt oder schwierige Behandlungssituationen in Rollenspielen durchgespielt (• Tab. 1).

Alle Teilnehmer erhielten zu den unterschiedlichen Themengebieten Grundlagenliteratur, zu denen sie außerdem ergänzende wissenschaftliche Publikationen selbstständig recherchieren und vorstellen konnten. Die Art der Gruppenarbeit und die Gestaltung des Referats setzten die Teilnehmer*innen selbstständig um. Bei Bedarf ergänzte das Organisationsteam relevante Punkte. Die Benotung der Teilnehmer*innen setzte sich aus dem Referat, Umsetzung der Gruppenarbeit und Engagement während der Veranstaltung zusammen.

Am dritten Tag der Lehrveranstaltung erhielten die Studierenden Einblicke in die Bereiche der Physiotherapie und Logopädie. Neben der Darstellung der Therapieoptionen beider Bereiche, wurden auch verschiedene Krankheitsbilder im Rahmen dessen besprochen. Die Teilnehmer*innen führten unter Anleitung selbst physikalische und logopädische Übungen durch (• Abb. 1).

Da auch die Neurologie bei rheumatischen Patienten eine große Rolle spielt, wurde dies am nächsten Tag thematisiert. Die Abläufe einer neurologischen

Z Rheumatol 2020 · 79:200-202 https://doi.org/10.1007/s00393-020-00749-8

(c) Der/die Autor(en) 2020

R. Hasseli · S. Pfeiffer · J. Kappesser · C. Hermann - K. Richter-Bastian - T. Sattler · M. Tschernatsch · U. Hoffmann · U. Müller-Ladner · U. Lange

\section{Modellprojekt zur interdisziplinären universitären Lehre - Studierende der Medizin und der Psychologie lernen erstmals gemeinsam}

\section{Zusammenfassung}

Eine interdisziplinäre Zusammenarbeit ist in der medizinischen Versorgung chronisch erkrankter Patienten mit komplexen Erkrankungen erforderlich. Vor allem im Bereich der internistischen Rheumatologie ist eine interdisziplinäre Arbeit unerlässlich, um die komplexen somatischen und psychosozialen Aspekte einer chronischen Erkrankung zu berücksichtigen. Dennoch werden die Aspekte der interprofessionellen Arbeit im Studium der Medizin und Psychologie unzureichend thematisiert. Aus diesem Grund wurde ein Modellprojekt zur interdisziplinären universitären Lehre konzipiert, welches beide Fächer miteinander vereint. Die Veranstaltung wurde im Wintersemester 2019/20 erstmalig durchgeführt und stieß bei den Teilnehmer*innen auf positive Resonanz. Das Hauptziel der Veranstaltung ist die Implementierung interprofessioneller Arbeit in die Ausbildung des medizinischen Personals. Zusätzlich konnte das Fach der internistischen Rheumatologie den Studierenden nähergebracht werden.

Schlüsselwörter

Interprofessionelle Lehre · Psychotherapie . Physiotherapie $\cdot$ Neurologie $\cdot$ Rheumatologie

\section{Model project on interdisciplinary university teaching-medical and psychology students learn together for the first time}

\section{Abstract}

An interdisciplinary collaboration is required in the medical care of chronically ill patients with complex illnesses. Especially in the field of internistic rheumatology, interdisciplinary work is essential to consider the complex somatic and psychosocial aspects of a chronic illness. Nevertheless, the aspects of interprofessional work in the study of medicine and psychology are insufficiently addressed. For this reason, a model project for interdisciplinary university teaching was conceived, which combines both subjects. The course was held for the first time in semester 2019/2020 and was rated excellent by the participants. The main goal of the course is the implementation of interprofessional work in the training of medical personnel. In addition, the discipline of internistic rheumatology could be brought closer to the students.

\section{Keywords}

Interprofessional training · Psychotherapy . Physiotherapy · Neurology · Rheumatology
Untersuchung wurden am Patienten demonstriert. Zudem wurden verschiedene Schmerzqualitäten und ihre Genese besprochen.

Um die erarbeiteten Inhalte auch in der Praxis zu erproben, durften die Teilnehmer*innen in gemischten 2er-Gruppen Patienten anamnestizieren, untersuchen und gemeinsam Problemlösungen erarbeiten. Die Teilnehmer ${ }^{\star}$ innen konnten somit Einblicke in die Herangehensweise der jeweils anderen Profession erhalten. Hierbei konnten Schwächen und Stärken beider Professionen dargestellt und behoben werden.
Akquiriert wurden die Studierenden über die digitale universitäre Lehrangebotsplattform für den Fachbereich der Medizin und Psychologie. Da es sich um eine Blockveranstaltung über 1 Woche handelt, wurde das Lehrkonzept in der vorlesungsfreien Zeit angeboten. Trotz der Veranstaltung in diesem Zeitraum war die Bewerberquote höher als die verfügbaren Plätze, was das große Interesse an diesem Modellprojekt unterstreicht. Beide Studiengänge sind unterschiedlich aufgebaut. Im Studiengang der Psychologie, der auf einem Bachelor-/ Masterstudiengang basiert, werden die Lehrveranstaltungen mit Credit-Points 


\section{Tab. 1 Zeitplan des Lehrkonzepts}

Tag 1 Referatsthemen (jeweils 45 minje Thema)

und 2 1. Rheumatische Erkrankungen \& Schmerzen: Einführung \& Überblick

2. Erstdiagnose: akute Erkrankung \& psycholog. Anamnese \& Diagnostik

3. Chronische Erkrankung: psychologische \& medikamentöse Therapie von Schmerzen

4. Einfluss psychosozialer Faktoren auf Krankheitsverlauf \& therapeutische Strategien

Gruppenarbeit, z. B. (jeweils $45 \mathrm{~min}$ ):

Darstellung von schwierigen Behandlungssituationen

Imaginationsübungen

Fallbearbeitungen

Interaktive Fragerunde zu Punkten der Referatsthemen

Tag 3 Grundlagen der Physiotherapie

Verschiedene Aspekte der Physiotherapie

Studienlage physikalischer Therapien

Physiotherapie in Eigenerfahrung

Rundgang durch die Räume der physikalischen Therapie zur Darstellung der Möglich-

keiten

Grundlagen der Logopädie

Verschiedene Aspekte der Logopädie

Therapiemöglichkeiten abhängig von Krankheitsbild

Koordination der Schlund- und mimischen Muskulatur

Logopädische Übungen in Eigenerfahrung

Tag 4 Neurologie und Schmerztherapie

Neurologische Erkrankungen

Neurologische Untersuchung

Schmerzwahrnehmung abhängig von Genese

Praxis am Patienten

Aufteilung der Studierenden in 2er-Gruppen

Anwenden des theoretischen Wissens am Patienten unter Supervision

Anamnese, Befunderhebung, Erstellen eines möglichen Therapiekonzepts

(CP) bewertet. Aus diesem Grund musste das Lehrkonzept derartig konzipiert werden, dass dafür $6 \mathrm{CP}$ vergeben werden konnten. Eine weitere Hürde war, dass beide Studiengänge später zwar interprofessionell arbeiten, aber bislang keine Verknüpfung während des Studiums stattfindet. Aus diesem Grund mussten auch Ressentiments bei den jeweiligen Fachbereichen beseitigt werden, was das Organisationsteam sehr gut umsetzen konnte.

Ziel dieses Lehrkonzepts ist es, neben der frühen Sensibilisierung für chronisch erkrankte Patienten auch die Bedeutung der interdisziplinären Zusammenarbeit hervorzuheben. Mithilfe des Lehrkonzepts erfolgte nicht nur eine Wissensvermittlung verschiedener medizinischer Professionen, sondern es konnten auch Berührungsängste der Teilnehmer aus den verschiedenen Fachdisziplinen abgebaut werden.

Als Nebeneffekt wurde den Teilnehmern das Fach der internistischen Rheumatologie deutlich nähergebracht. Längerfristig wird dies sicherlich zu einer besseren Präsenz der Rheumatologie so- wohl bei Medizin- wie auch den Psychologiestudierenden führen. Wir hoffen, dass unser Konzept auch an anderen Universitäten Interesse weckt und ggf. auch an anderen Standorten umgesetzt wird. Gerne stehen die Autoren bei der Umsetzung mit Rat und Tat zur Seite.

\section{Fazit für die Praxis}

- Eine interprofessionelle Patientenversorgung verbessert den Krankheitsverlauf (u.a. Therapieadhärenz, Krankheitsbewältigung und Compliance) der Patienten und die Teamarbeit des medizinischen Personals.

- Bereits die Ausbildung des medizinischen Personals sollte interprofessionell gestaltet werden.

- Ein erster Schritt für eine interprofessionelle Lehre zwischen den Fachbereichen Humanmedizin und Psychologie konnte erfolgreich an der Universität Gießen umgesetzt werden.

- Diese interprofessionelle Lehre soll auch in den nächsten Jahren weiter optimiert und umgesetzt werden

\section{Korrespondenzadresse}

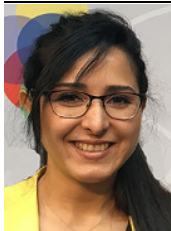

Dr. med. R. Hasseli

Abteilung für Rheumatologie und Klinische Immunologie, Justus-Liebig-Universität Gießen, Campus Kerckhoff Benekestr. 2-8, $61231 \mathrm{Bad}$ Nauheim, Deutschland r.hasseli@kerckhoff-klinik.de

Funding. Open Access funding provided by Projekt DEAL.

\section{Einhaltung ethischer Richtlinien}

Interessenkonflikt. R. Hasseli, S. Pfeiffer, J. Kappesser, C. Hermann, K. Richter-Bastian, T. Sattler, M. Tschernatsch, U. Hoffmann, U. Müller-Ladner und U. Lange geben an, dass kein Interessenkonflikt besteht.

Für diesen Beitrag wurden von den Autoren keine Studien an Menschen oder Tieren durchgeführt. Für die aufgeführten Studien gelten die jeweils dort angegebenen ethischen Richtlinien.

Open Access. Dieser Artikel wird unter der Creative Commons Namensnennung 4.0 International Lizenz veröffentlicht, welche die Nutzung, Vervielfältigung Bearbeitung, Verbreitung und Wiedergabe in jeglichem Medium und Format erlaubt, sofern Sie den/die ursprünglichen Autor(en) und die Quelle ordnungsgemäß nennen, einen Link zur Creative Commons Lizenz beifügen und angeben, ob Änderungen vorgenommen wurden.

Die in diesem Artikel enthaltenen Bilder und sonstiges Drittmaterial unterliegen ebenfalls der genannten Creative Commons Lizenz, sofern sich aus der Abbildungslegende nichts anderes ergibt. Sofern das betreffende Material nicht unter der genannten Creative Commons Lizenz steht und die betreffende Handlung nicht nach gesetzlichen Vorschriften erlaubt ist, ist für die oben aufgeführten Weiterverwendungen des Materials die Einwilligung des jeweiligen Rechteinhabers einzuholen.

Weitere Details zur Lizenz entnehmen Sie bitte der Lizenzinformation auf http://creativecommons.org/ licenses/by/4.0/deed.de. 